\title{
Long-term association of food and nutrient intakes with cognitive and functional decline: a 13-year follow-up study of elderly French women
}

\author{
Marie-Noël Vercambre ${ }^{1,2}$, Marie-Christine Boutron-Ruault ${ }^{1}$, Karen Ritchie ${ }^{2}$, Françoise Clavel-Chapelon ${ }^{1 *}$ \\ and Claudine Berr ${ }^{2}$ \\ ${ }^{1}$ INSERM (Institut National de la Santé et de la Recherche Médicale), ERI 20, EA 4045, and Institut Gustave Roussy, Villejuif \\ F-94805, France \\ ${ }^{2}$ INSERM, U888, Montpellier, F-34093 France; Univ Montpellierl, Montpellier F-34000, France
}

(Received 7 July 2008 - Revised 31 October 2008 - Accepted 21 November 2008 - First published online 10 February 2009)

The objective of the present study was to determine the potential long-term impact of dietary habits on age-related decline among 4809 elderly women (born between 1925 and 1930) in the 'Etude Epidémiologique de Femmes de la Mutuelle Générale de l'Education Nationale' (E3N) study, a French epidemiological cohort. In 1993, an extensive diet history self-administered questionnaire was sent to all participants, and in 2006 another questionnaire on instrumental activities of daily living (IADL) and recent cognitive change was sent to a close relative or friend of each woman. Logistic models adjusted for socio-demographic, lifestyle and health factors were performed to evaluate associations between habitual dietary intakes and two outcomes of interest based on the informant response: recent cognitive decline and IADL impairment. Recent cognitive decline was associated with lower intakes of poultry, fish, and animal fats, as well as higher intakes of dairy desserts and ice-cream. IADL impairment was associated with a lower intake of vegetables. The odds of recent cognitive decline increased significantly with decreasing intake of soluble dietary fibre and $n-3$ fatty acids but with increasing intake of retinol. The odds of IADL impairment increased significantly with decreasing intakes of vitamins $\mathrm{B}_{2}, \mathrm{~B}_{6}$ and $\mathrm{B}_{12}$. These results are consistent with a possible long-term neuroprotective effect of dietary fibre, $n$ - 3 polyunsaturated fats and $\mathrm{B}$-group vitamins, and support dietary intervention to prevent cognitive decline.

Ageing: Cognition: Dietary habits: Function: Longitudinal studies: Nutrition: Women

Age-related cognitive decline is an important public health concern, with a prevalence that is rapidly increasing with population ageing. Cognitive impairment leads to significant functional loss and is a major component of total age-related deterioration ${ }^{(1)}$. In the absence of curative treatments, prevention has become a real challenge. Several risk factors are known to be involved in the aetiology of cognitive impairment $^{(2)}$, but few are modifiable, among them nutrition. Longitudinal studies investigating dietary influence on cognitive outcomes in the elderly have suggested that increased intakes of specific vitamins or minerals could be associated with a reduced incidence of cognitive impairment ${ }^{(3)}$. To explain these results, different hypotheses involving oxidative stress, or inflammatory or vascular pathways have been put forward. However, the long-term relationship between diet and cognitive decline remains unclear and even conflicting. A better understanding of dietary factors that contribute to the maintenance of cognitive ability is thus of high priority ${ }^{(4)}$. The importance of prospective studies of specifically long duration, which would include subjects whose diet was monitored long before cognitive assessment, has previously been stressed ${ }^{(5)}$. Using longitudinal data from the 'Etude Epidémiologique de Femmes de la Mutuelle Générale de l'Education Nationale' (Epidemiological Study of Women of MGEN) (E3N) study, we examined the associations between previous usual diet and age-related cognitive impairment.

\section{Subjects and methods

\begin{abstract}
The 'Etude Epidémiologique de Femmes de la Mutuelle Générale de l'Education Nationale' cohort
\end{abstract}

The E3N cohort includes 98995 French women within the National Education System, born between 1925 and 1950. This ongoing prospective study primarily investigates cancer risk factors ${ }^{(6)}$ in women, with particular focus on diet and hormones. The research programme was approved by the Bicêtre Hospital Review Board and the French National Commission for Data Protection and Privacy. All study participants gave informed consent and, since June 1990, they have been asked at approximately 24-month intervals to complete

\footnotetext{
Abbreviations: DECO, Détérioration Cognitive Observée questionnaire; E3N, Etude Epidémiologique de Femmes de la Mutuelle Générale de l'Education Nationale; IADL, instrumental activities of daily living.

* Corresponding author: Dr Françoise Clavel-Chapelon, fax +331421140 00, email clavel@igr.fr
} 
self-administered questionnaires on medical events and a variety of lifestyle characteristics.

\section{The ageing sub-cohort of the Etude Epidémiologique de Femmes de la Mutuelle Générale de l'Education Nationale study}

In 2006, a specific ageing survey was carried out. Of the 98995 women in the cohort, those who where born between 1925 and 1930 ( $n$ 10040) represented the target population, because of the higher prevalence of cognitive impairment in this stratum than in middle-aged women. Among these 10040 elderly women, 1095 were deceased or had dropped out. Therefore, the ageing survey involved 8945 cohort participants. A questionnaire intended for a close relative or friend of the E3N participant was designed to obtain indirect data on cognitive and functional problems faced by the elderly women. The questionnaire was sent in January 2006. It included eight instrumental activities of daily living $(\mathrm{IADL})^{(7)}$ and the 'DEtérioration Cognitive Observée' (observed cognitive deterioration) (DECO) scale ${ }^{(8)}$. While IADL items provide a comprehensive view of the functional consequences of cognitive decline in everyday life (telephone use, shopping, mode of transportation, ability to handle personal medication, finance handling, food preparation, housekeeping, laundry), DECO provides more specific information on cognitive functioning observed by informants over the past year. This nineteen-item Likert scale allows the evaluation of recent cognitive decline through alterations of the capacity to perform specific tasks related to memory, attention, and visuospatial and language skills.

A questionnaire from a relative was obtained for 5941 participants $(66.4 \%)$. Functional and cognitive data were complete for a total of 5839 women. Because of missing or non-physiologically plausible dietary data - the exposure of interest, assessed in 1993 - the analysis sample finally included 4809 elderly women. Dietary data were considered as non-physiologically plausible when the calculated ratio of energy intake to energy requirement fell in the highest or lowest percentile for the entire cohort ${ }^{(9)}$.

\section{Outcomes of interest (2006)}

In order to explore the nutrition-cognition relationship, we examined two aspects of cognitive functioning, based on informant report: cognitive decline over a period of 1 year and cognitive status as reflected by current impact on everyday functioning.

Recent cognitive decline was defined on the basis of the DECO score (range 0-38), according to a threshold of 33 . This cut-off point has been previously shown to distinguish subjects with a high risk of progressive pathological decline in a French general population sample, with a sensitivity of $89 \%$ and a specificity of $67 \%(10)$. The individuals with a DECO score under 33 constituted the group of recent cognitive decliners.

The functional dimension of age-related decline was based on a simplified IADL scale, which has been previously validated ${ }^{(11)}$ : for each woman, we calculated the 4-IADL score by summing up the number of limitations to the subject's ability to use the telephone, take her medications, use public transport and manage her own budget. The women with a non-null 4-IADL score constituted the group of functional decliners (sensitivity of $62 \%$ and specificity of $80 \%$ for cognitive troubles).

\section{Dietary assessment (1993)}

Dietary data were collected in 1993 with an extensive diet history questionnaire covering daily consumption of 208 foods and beverages. The dietary questionnaire was sent with a booklet of photographs to facilitate estimation of portion sizes. Both the questionnaire and the illustrated booklet had been previously validated ${ }^{(12,13)}$ on a sample of 115 women, taking the average of twelve $24 \mathrm{~h}$ dietary recalls obtained at monthly intervals over a 1-year period as reference. Average daily dietary intakes of macro- and micronutrients were estimated based on dietary questionnaire data, and using a food composition table derived from the French national database $^{(14)}$. A high proportion of subjects $(76 \%$ for foods and $72 \%$ for nutrients on average) were classified in the same or adjacent quintiles for the dietary questionnaire and $24 \mathrm{~h}$ recalls. Among the analysis sample, mean age at dietary assessment was 65.5 (SD 1.8) years.

\section{Potential confounders}

Socio-economic factors, lifestyle and medical background have been associated with cognitive ageing ${ }^{(15)}$. To limit confounding effects in analyses, adjustment variables included socio-demographic characteristics (age in 2006 as 76-79v. $80-82$ years; education level as $<12$ years $v$. $\geq 12$ years), BMI $\quad\left(<18.5 \mathrm{~kg} / \mathrm{m}^{2} ; \quad 18.5-24.9 \mathrm{~kg} / \mathrm{m}^{2} ; \quad 25-29.9 \mathrm{~kg} / \mathrm{m}^{2}\right.$; $\geq 30 \mathrm{~kg} / \mathrm{m}^{2}$ ), an indicator of average physical activity based on the median value in metabolic equivalents per week $(\leq 50 ;>50)$, the quartiles of average dietary energy intake in $\mathrm{kJ} / \mathrm{d}$, as well as smoking status (current or past smoker; non-smoker). Supplement consumption was taken into account (use of vitamin $\mathrm{D}$ and/or $\mathrm{Ca}$, use of other vitamins or minerals). Other self-reported variables related to medical follow-up were use of postmenopausal hormones (ever; never), diabetes mellitus (ever; never), hypertension (ever; never) and hypercholesterolaemia (ever; never). Adjustment variables also included self-reported history of CHD (myocardial infarction or angina pectoris), stroke, cancer and depression. Data on cancer were validated through pathological reports, as the main outcome of the E3N study.

\section{Missing values}

Missing values for educational level, BMI, supplement consumption, physical activity and smoking status represented less than $5 \%$ of subjects for each of these covariates. Thus, we replaced them by the modal value. This procedure is routinely used when analysing E3N data for adjustment categorical variables, in order to include all subjects with non-missing data for the main outcomes/exposures, so as to limit bias and power loss due to too many excluded cases. However, we verified that results of statistical models were globally unchanged when subjects with one or more missing values for any of these variables were excluded from the analyses (restricted sample of 4515 subjects). 


\section{Statistical analyses}

To illustrate the link between cognitive and functional status as appraised by the informant, we performed kernel density estimation $^{(16)}$ of the DECO score according to the 4-IADL score. This analysis was conducted among the 4758 women for whom both scores were computable. We studied bivariate associations between potential confounders and the two outcomes of interest: DECO score $<33$ and 4-IADL score $>0$. All variables listed above as potential confounders were then included in models assessing nutritional factors.

Dietary habits were approached by considering successively daily intakes of food groups (for example, vegetables, fish, eggs), macronutrients (for example, carbohydrates, proteins) and micronutrients (for example, vitamins and minerals). Raw intakes of food groups as well as residuals on energy ${ }^{(17)}$ of nutrient intakes were categorised into tertiles according to the distribution in our elderly population, except when food groups were consumed by less than $10 \%$ of the sample. In those cases (i.e. legumes; pizza, sandwiches and snacks; beef, pork and lamb; poultry; offal; animal fats; dairy desserts and ice-cream; sugar and confectionery; pastries and cakes; coffee; tea; soups; wine; beer; other alcoholic drinks), we isolated a category of non-consumers, and considered consumers in two groups according to the median consumption. Although alcohol is a nutrient, it was categorised as described above for rarely consumed food groups, since $13.8 \%$ of women in our sample showed null intake.

We used multivariable logistic regression to compute the OR and $95 \%$ CI. Each nutritional factor considered was entered into separate logistic regression models. Tests for linear trend were performed using the ordinal score on categories of nutritional intake.

Except for the kernel density estimation, which was computed with R software version 2.3.0 (http://www.r-project.org), all analyses were performed using SAS software (version 9.1; SAS Institute, Inc., Cary, NC, USA). All results were considered significant at the $5 \%$ level. All statistical tests were two-sided.

\section{Results}

\section{Sample selection}

Compared with women for whom no relative responded, women for whom informant data were obtained in 2006 were somewhat younger and more educated. They were also more likely to be married and to have responded to the dietary questionnaire sent in 1993. In addition, women excluded from the analysis sample because of missing dietary data were less educated than the investigated population.

\section{Characteristics of women according to their cognitive and functional status}

Among the 4758 women included in the analysis sample, 518 women had a DECO score $<33(12.4 \%)$, 716 had a 4-IADL score $>0(14.9 \%)$ and 268 presented both declines $(5.6 \%)$. Although non-interchangeable, functional status and recent cognitive decline were closely related in our sample, as illustrated by Fig. 1, which simultaneously represents DECO score distributions in subjects with the 4-IADL score respectively equal to $0,1,2,3$ or 4 . While the maximum score on the DECO scale is 38 and the minimum is 0 (with lower scores

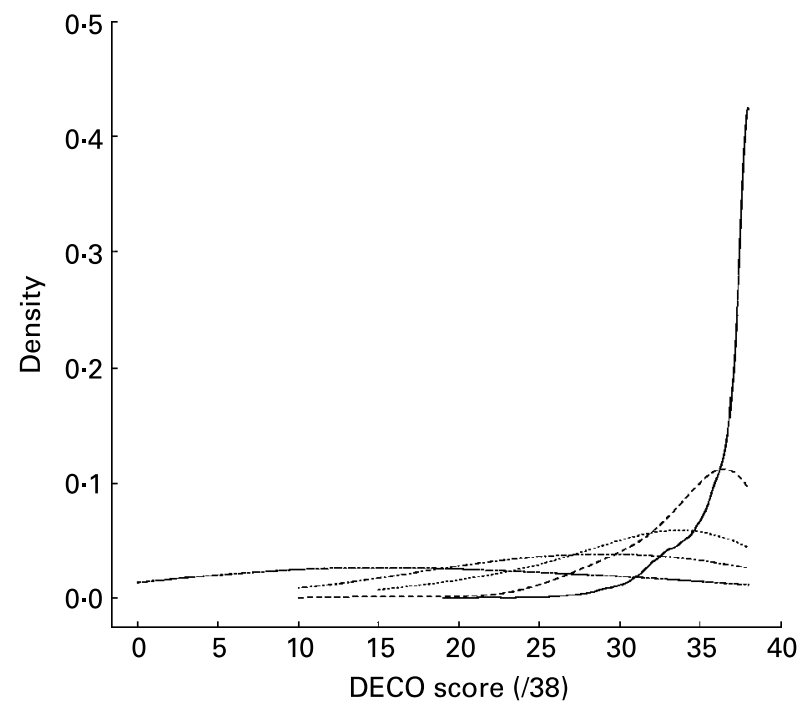

Fig. 1. Cognitive decline and functional impairment. Kernel density estimation of the 'Détérioration Cognitive Observée' (observed cognitive deterioration) questionnaire (DECO) score according to the Instrumental Activities of Daily Living (IADL) score in the 'Etude Epidémiologique de Femmes de la Mutuelle Générale de l'Education Nationale' (E3N) study, France, 2006. The 4-IADL score is defined as the number of limitations to the subject's ability to use the telephone, take medication, use public transport and manage her own budget. The analysis was conducted among the 4758 subjects for whom both scores were computable. (-), 4-IADL score $=0$ ( $n$ 4093); $(---)$, 4-IADL score = 1 ( $n$ 493); (---), 4-IADL score = 2 ( $n$ 88); (-..-), 4-IADL score $=3$ (n 35); (.-.-.), 4-IADL score $=4$ ( $n$ 49).

indicating a sharper decrease in cognitive performance), DECO cut-off points for the first, second and third quartiles were, respectively: 36,38 and 38 for women without disability; 32, 35 and 37 for women who had one IADL impairment; 28, 33 and 36 for women who had two IADL impairments; 23, 27 and 34 for women who had three IADL impairments; 10, 17 and 28 for women who had four IADL impairments.

Characteristics of women as a function of their cognitive and functional status are described in Table 1. Recent cognitive decline was positively associated with age, history of depression, cancer, CHD, stroke and diabetes mellitus, but inversely associated with education level and physical activity level. IADL impairment was associated with higher age, lower education level, BMI $\geq 25 \mathrm{~kg} / \mathrm{m}^{2}$, lower energy intake, neversmoker status, lower level of physical activity, non-use of supplements and non-use of postmenopausal hormones. IADL impairment was also positively associated with history of depression, CHD, stroke, diabetes mellitus and hypertension.

\section{Dietary intakes and age-related decline}

Results of multi-adjusted logistic models that tested associations between age-related decline and habitual dietary intakes of twenty-five food-groups are shown in Table 2. After controlling for various potential factors, women with recent cognitive decline consumed in the past significantly lower amounts of poultry, fish, and animal fats. They also consumed higher amounts of dairy desserts and ice-cream. The association between higher odds of cognitive decline and higher intake of pastries and cakes was borderline significant. Women with IADL impairment had lower intake of vegetables. 
Table 1. Comparison of elderly women ( $n$ 4809) of the 'Etude Epidémiologique de Femmes de la Mutuelle Générale de l'Education Nationale' (E3N) study, France, 1993-2006, depending on their cognitive and functional status

\begin{tabular}{|c|c|c|c|c|c|c|}
\hline \multirow[b]{2}{*}{ Covariate } & \multicolumn{3}{|c|}{ Recent cognitive decline (DECO score $<33$ ) } & \multicolumn{3}{|c|}{ Functional impairment (4-IADL score $>0$ ) } \\
\hline & Non-cases $(n$ 4211) (\%) & Cases $(n 598)(\%)$ & $P^{\star}$ & Non-cases ( $n$ 4093) (\%) & Cases $(n 716)(\%)$ & $P^{\star}$ \\
\hline \multicolumn{7}{|c|}{ Age at the time of cognitive assessment (2006) } \\
\hline $76-79$ years & $78 \cdot 8$ & $75 \cdot 1$ & & $79 \cdot 1$ & 74.2 & \\
\hline $80-82$ years & $21 \cdot 2$ & 24.9 & 0.037 & $20 \cdot 9$ & $25 \cdot 8$ & 0.003 \\
\hline \multicolumn{7}{|l|}{ Education level† } \\
\hline$<12$ years & $15 \cdot 6$ & 21.9 & & $15 \cdot 1$ & $23 \cdot 6$ & \\
\hline$\geq 12$ years & 84.4 & $78 \cdot 1$ & 0.000 & 84.9 & $76 \cdot 4$ & 0.000 \\
\hline \multicolumn{7}{|l|}{ BMI $\left(\mathrm{kg} / \mathrm{m}^{2}\right) \dagger$} \\
\hline$<18.5$ & $2 \cdot 8$ & 4.0 & 0.114 & 2.9 & 3.4 & 0.312 \\
\hline $18.5-24.9$ & $72 \cdot 5$ & $71 \cdot 1$ & & $73 \cdot 3$ & $66 \cdot 5$ & \\
\hline $25 \cdot 0-29 \cdot 9$ & $21 \cdot 2$ & $22 \cdot 2$ & 0.523 & $21 \cdot 0$ & $23 \cdot 2$ & 0.044 \\
\hline$\geq 30.0$ & 3.5 & $2 \cdot 7$ & 0.345 & $2 \cdot 8$ & $7 \cdot 0$ & 0.000 \\
\hline \multicolumn{7}{|c|}{ Indicator of average physical activity (metabolic equivalents per week) } \\
\hline$\leq 50$ & $49 \cdot 3$ & 53.8 & & $48 \cdot 6$ & $57 \cdot 1$ & \\
\hline$>50$ & $50 \cdot 7$ & $46 \cdot 2$ & 0.039 & 51.4 & $42 \cdot 9$ & 0.000 \\
\hline \multicolumn{7}{|c|}{ Average daily energy intake $(\mathrm{kJ} / \mathrm{d}) \ddagger$} \\
\hline Energy $\leq 6276$ & $23 \cdot 1$ & $24 \cdot 6$ & 0.370 & $22 \cdot 5$ & $27 \cdot 7$ & 0.007 \\
\hline $6276<$ energy $\leq 7950$ & $24 \cdot 1$ & $22 \cdot 9$ & & $24 \cdot 3$ & $21 \cdot 8$ & \\
\hline $7950<$ energy $\leq 9623$ & $26 \cdot 9$ & $24 \cdot 2$ & 0.675 & $26 \cdot 9$ & 24.7 & 0.825 \\
\hline Energy $>9623$ & $25 \cdot 9$ & $28 \cdot 3$ & 0.263 & $26 \cdot 3$ & $25 \cdot 8$ & 0.422 \\
\hline \multicolumn{7}{|l|}{ Smoking status $†$} \\
\hline Never-smoker & $66 \cdot 5$ & 69.7 & & $66 \cdot 3$ & $70 \cdot 3$ & \\
\hline Smoker or past smoker & 33.5 & $30 \cdot 3$ & 0.118 & 33.7 & $29 \cdot 7$ & 0.040 \\
\hline \multicolumn{7}{|c|}{ Supplement of vitamin D and/or Ca† } \\
\hline Non-use & 83.8 & $84 \cdot 6$ & & $83 \cdot 8$ & $84 \cdot 4$ & \\
\hline Use & $16 \cdot 2$ & $15 \cdot 4$ & 0.614 & $16 \cdot 2$ & $15 \cdot 6$ & 0.724 \\
\hline \multicolumn{7}{|c|}{ Supplement of other vitamins or minerals $\dagger$} \\
\hline Non-use & $80 \cdot 0$ & $77 \cdot 3$ & & $79 \cdot 1$ & $83 \cdot 0$ & \\
\hline Use & $20 \cdot 0$ & $22 \cdot 7$ & 0.119 & $20 \cdot 9$ & $17 \cdot 0$ & 0.018 \\
\hline \multicolumn{7}{|c|}{ Use of postmenopausal hormones } \\
\hline Never & $50 \cdot 4$ & 51.5 & & 49.5 & $56 \cdot 4$ & \\
\hline Ever & $49 \cdot 6$ & 48.5 & 0.618 & $50 \cdot 5$ & $43 \cdot 6$ & 0.001 \\
\hline \multicolumn{7}{|l|}{ History of depression } \\
\hline Never & $80 \cdot 2$ & $69 \cdot 6$ & & $80 \cdot 2$ & 71.4 & \\
\hline Ever & $19 \cdot 8$ & $30 \cdot 4$ & 0.000 & $19 \cdot 8$ & $28 \cdot 6$ & 0.000 \\
\hline \multicolumn{7}{|l|}{ History of cancer } \\
\hline Never & $84 \cdot 2$ & $80 \cdot 8$ & & 83.9 & 83.0 & \\
\hline Ever & $15 \cdot 8$ & $19 \cdot 2$ & 0.036 & $16 \cdot 1$ & $17 \cdot 0$ & 0.541 \\
\hline \multicolumn{7}{|l|}{ History of $\mathrm{CHD \S}$} \\
\hline Never & $94 \cdot 2$ & $90 \cdot 3$ & & $94 \cdot 2$ & $90 \cdot 6$ & \\
\hline Ever & $5 \cdot 8$ & $9 \cdot 7$ & 0.000 & $5 \cdot 8$ & 9.4 & 0.000 \\
\hline \multicolumn{7}{|l|}{ History of stroke } \\
\hline Never & $96 \cdot 3$ & $93 \cdot 0$ & & $96 \cdot 6$ & $91 \cdot 8$ & \\
\hline Ever & 3.7 & $7 \cdot 0$ & 0.000 & 3.4 & 8.2 & 0.000 \\
\hline \multicolumn{7}{|l|}{ History of diabetes mellitus } \\
\hline Never & $94 \cdot 2$ & $90 \cdot 6$ & & 94.5 & 89.5 & \\
\hline Ever & $5 \cdot 8$ & $9 \cdot 4$ & 0.001 & 5.5 & 10.5 & 0.000 \\
\hline \multicolumn{7}{|l|}{ History of hypertension } \\
\hline Never & $56 \cdot 5$ & $57 \cdot 9$ & & $57 \cdot 4$ & $52 \cdot 7$ & \\
\hline Ever & 43.5 & $42 \cdot 1$ & 0.543 & $42 \cdot 6$ & $47 \cdot 3$ & 0.018 \\
\hline \multicolumn{7}{|c|}{ History of hypercholesterolaemia } \\
\hline Never & $57 \cdot 6$ & $56 \cdot 5$ & & 57.5 & $57 \cdot 4$ & \\
\hline Ever & 42.4 & 43.5 & 0.621 & 42.5 & $42 \cdot 6$ & 0.975 \\
\hline
\end{tabular}

DECO, 'Détérioration Cognitive Observée' (observed cognitive deterioration) questionnaire; IADL, instrumental activities of daily living.

${ }^{*} P$ values obtained through univariate logistic regression analyses.

† Missing values were replaced by the most frequent modality (variables with less than $5 \%$ missing values).

$\ddagger$ Except energy from alcohol.

$\S$ Myocardial infarction or angina pectoris.

Results of multi-adjusted logistic models that tested associations between age-related decline and habitual dietary intakes of thirty-three macro- or micronutrients are shown in Table 3. The odds of recent cognitive decline increased with decreasing intake of total dietary fibre, soluble dietary fibre and of $n-3$ fatty acids, while they increased with increasing intake of retinol. Furthermore, recent cognitive decliners showed a higher $n-6: n-3$ fatty acid ratio than women with a DECO score $\geq 33$. Concerning functional status, the odds of IADL impairment increased significantly with decreasing intake of vitamin $\mathrm{B}_{2}, \mathrm{~B}_{6}$ and $\mathrm{B}_{12}$. 
Table 2. Multi-adjusted odds ratios of habitual dietary habits (assessed in 1993) associated with cognitive decline or instrumental activities of daily living (IADL) impairment (assessed in 2006) in elderly women ( $n$ 4809) of the 'Etude Epidémiologique de Femmes de la Mutuelle Générale de l'Education Nationale' (E3N) study, France, 1993-2006*

\begin{tabular}{|c|c|c|c|c|c|c|c|c|c|c|c|c|}
\hline \multirow[b]{3}{*}{ Food group intake (g/d) } & \multirow[b]{3}{*}{ Mean‡ } & \multirow[b]{3}{*}{ SD $\ddagger$} & \multicolumn{5}{|c|}{ Recent cognitive decline (DECO score $<33)(n 598)$} & \multicolumn{5}{|c|}{ Functional impairment $(4-I A D L$ score $>0)(n 716)$} \\
\hline & & & \multicolumn{2}{|c|}{ Group 2 v. group $1 \dagger$} & \multicolumn{2}{|c|}{ Group 3 v. group $1 \dagger$} & \multirow[b]{2}{*}{$P$ for trend } & \multicolumn{2}{|c|}{ Group 2 v. group $1 \dagger$} & \multicolumn{2}{|c|}{ Group 3 v. group 1† } & \multirow[b]{2}{*}{$P$ for trend } \\
\hline & & & OR & $95 \% \mathrm{Cl}$ & OR & $95 \% \mathrm{Cl}$ & & OR & $95 \% \mathrm{Cl}$ & OR & $95 \% \mathrm{Cl}$ & \\
\hline Potatoes & $65 \cdot 82$ & 55.01 & 1.01 & $0.81,1.25$ & 0.90 & $0.72,1.13$ & 0.377 & 1.07 & $0.87,1.31$ & 0.95 & $0.77,1.18$ & 0.646 \\
\hline Vegetables & $231 \cdot 7$ & $118 \cdot 7$ & $1 \cdot 12$ & $0.90,1.39$ & $1 \cdot 10$ & $0.89,1.37$ & 0.389 & 0.83 & $0.68,1.01$ & 0.80 & $0.65,0.98$ & 0.029 \\
\hline Legumes§ & 14.89 & 19.89 & 0.89 & $0.71,1 \cdot 11$ & 1.03 & $0.82,1.29$ & 0.700 & 0.92 & $0.75,1 \cdot 14$ & 0.91 & $0 \cdot 74,1 \cdot 13$ & 0.405 \\
\hline Fruits and fruit juice & 354.5 & $199 \cdot 4$ & 0.89 & $0.72,1 \cdot 11$ & 0.88 & $0.70,1.09$ & 0.240 & 0.94 & $0.77,1 \cdot 15$ & 0.86 & $0.70,1.06$ & $0 \cdot 164$ \\
\hline Milk and yoghurt & 234.8 & 191.9 & $1 \cdot 21$ & $0.97,1.50$ & $1 \cdot 17$ & $0.93,1.46$ & 0.182 & 1.04 & $0.85,1.26$ & 0.97 & $0.79,1 \cdot 20$ & 0.799 \\
\hline Cheese & $48 \cdot 78$ & $39 \cdot 16$ & $1 \cdot 11$ & $0.89,1.39$ & $1 \cdot 15$ & $0.92,1.44$ & 0.223 & 1.04 & $0.85,1 \cdot 28$ & 1.00 & $0.82,1 \cdot 24$ & 0.974 \\
\hline Bread and cereal products & $131 \cdot 6$ & 85.59 & 0.91 & $0.72,1 \cdot 14$ & 1.00 & $0.78,1.27$ & 0.997 & 0.91 & $0.74,1.13$ & 1.02 & $0.81,1 \cdot 28$ & 0.902 \\
\hline Pizza, sandwiches and snacks§ & 19.98 & 22.56 & 0.91 & $0.69,1 \cdot 22$ & 0.95 & $0.71,1.27$ & 0.947 & $0 \cdot 81$ & $0.62,1.04$ & 0.85 & $0 \cdot 66,1 \cdot 11$ & 0.523 \\
\hline Beef, pork and lamb§ & 45.45 & 35.53 & 0.99 & $0 \cdot 76,1 \cdot 30$ & 0.87 & $0.66,1.15$ & 0.206 & 0.93 & $0.73,1.20$ & 0.96 & $0.74,1 \cdot 23$ & 0.873 \\
\hline Poultry§ & 16.93 & 17.89 & 0.88 & $0.71,1.09$ & 0.73 & $0.58,0.91$ & 0.004 & 0.82 & $0.67,1.00$ & 0.85 & $0.70,1.04$ & 0.136 \\
\hline Processed meat & $22 \cdot 12$ & $19 \cdot 26$ & $1 \cdot 13$ & $0.91,1.41$ & $1 \cdot 16$ & $0.92,1.46$ & 0.205 & 0.96 & $0.79,1 \cdot 18$ & 1.06 & $0 \cdot 86,1 \cdot 31$ & 0.596 \\
\hline Offal§ & $6 \cdot 34$ & 9.96 & 1.07 & $0.87,1.32$ & 1.01 & $0.81,1.25$ & 0.899 & 1.06 & $0.87,1.28$ & 0.90 & $0 \cdot 74,1 \cdot 10$ & 0.376 \\
\hline Fish & 38.03 & 28.45 & 0.88 & $0.71,1.08$ & 0.80 & $0.64,0.99$ & 0.043 & 0.87 & $0.71,1.06$ & 0.99 & $0.81,1 \cdot 21$ & 0.939 \\
\hline Eggs & $22 \cdot 20$ & 21.48 & 0.98 & $0.79,1 \cdot 22$ & 0.99 & $0.79,1.23$ & 0.897 & 0.99 & $0.81,1 \cdot 21$ & 1.06 & $0.86,1 \cdot 30$ & 0.584 \\
\hline Vegetable fats & $20 \cdot 37$ & $10 \cdot 76$ & 0.95 & $0 \cdot 76,1 \cdot 18$ & 1.02 & $0.82,1 \cdot 27$ & 0.842 & 1.03 & $0.84,1 \cdot 26$ & 1.02 & $0 \cdot 83,1 \cdot 26$ & 0.860 \\
\hline Animal fats§ & $8 \cdot 67$ & 9.97 & 0.94 & $0.75,1 \cdot 17$ & 0.71 & $0.56,0.90$ & 0.003 & 0.82 & $0.66,1.01$ & 0.81 & $0.65,1.01$ & 0.083 \\
\hline Dairy desserts and ice-cream§ & $22 \cdot 89$ & 35.78 & 1.02 & $0.82,1 \cdot 28$ & 1.33 & $1.07,1.65$ & 0.010 & 0.97 & $0.80,1.19$ & 1.06 & $0.86,1 \cdot 29$ & 0.612 \\
\hline Sugar and confectionery§ & 34.03 & 29.75 & 0.95 & $0 \cdot 70,1 \cdot 28$ & 0.96 & $0.70,1.31$ & 0.885 & 0.92 & $0 \cdot 70,1 \cdot 21$ & 0.95 & $0.72,1.27$ & 0.924 \\
\hline Pastries and cakes§ & 33.56 & 32.38 & $1 \cdot 10$ & $0.79,1.54$ & 1.29 & $0.92,1.81$ & 0.056 & 0.94 & $0.70,1.25$ & 0.99 & $0.74,1.34$ & 0.764 \\
\hline Coffee§ & $237 \cdot 2$ & $212 \cdot 5$ & 0.95 & $0.71,1 \cdot 27$ & 0.95 & $0.71,1.28$ & 0.804 & $1 \cdot 19$ & $0.89,1.59$ & $1 \cdot 12$ & $0.84,1 \cdot 50$ & 0.837 \\
\hline Tea§ & $169 \cdot 1$ & $270 \cdot 0$ & 1.08 & $0.87,1.33$ & 0.96 & $0.78,1.19$ & 0.781 & 0.89 & $0.73,1.08$ & 0.90 & $0.74,1.09$ & 0.248 \\
\hline Soups§ & $151 \cdot 4$ & $125 \cdot 0$ & $1 \cdot 17$ & $0.87,1.57$ & 1.07 & $0.79,1.45$ & 0.938 & 0.98 & $0 \cdot 75,1 \cdot 27$ & 0.93 & $0 \cdot 71,1 \cdot 22$ & 0.548 \\
\hline Beer§ & $11 \cdot 31$ & 47.67 & 1.04 & $0.78,1.38$ & 0.86 & $0 \cdot 63,1 \cdot 18$ & 0.459 & 1.08 & $0.82,1.42$ & $1 \cdot 19$ & $0.91,1.56$ & 0.175 \\
\hline Wine§ & 91.44 & $124 \cdot 3$ & 1.01 & $0.80,1.26$ & 0.94 & $0.75,1.18$ & 0.556 & 0.90 & $0.73,1.11$ & 0.85 & $0.68,1.04$ & $0 \cdot 123$ \\
\hline $\begin{array}{l}\text { Alcoholic drinks§ (other than } \\
\text { beer and wine) }\end{array}$ & $15 \cdot 25$ & $42 \cdot 11$ & 0.87 & $0.70,1.08$ & 0.96 & $0.77,1.20$ & 0.774 & $0 \cdot 85$ & $0.69,1.04$ & 0.82 & $0.66,1.01$ & 0.061 \\
\hline
\end{tabular}

DECO, 'Détérioration Cognitive Observée' (observed cognitive deterioration) questionnaire.

group. Adjustment was made on all covariates listed in Table 1. Each food group was considered separately.

† Group 1, group 2 and group 3 were defined as tertile 1, tertile 2 and tertile 3 of food intake unless otherwise indicated.

列 
Table 3. Multi-adjusted odds ratios of habitual nutrient intakes (assessed in 1993) associated with cognitive decline or instrumental activities of daily living (IADL) impairment (assessed in 2006) in elderly women ( $n$ 4809) of the 'Etude Epidémiologique de Femmes de la Mutuelle Générale de l'Education Nationale' (E3N) study, France, 1993-2006*

\begin{tabular}{|c|c|c|c|c|c|c|c|c|c|c|c|c|}
\hline \multirow[b]{3}{*}{ Nutrient intake (per d) } & \multirow[b]{3}{*}{ Meanł } & \multirow[b]{3}{*}{ SD $\ddagger$} & \multicolumn{5}{|c|}{ Recent cognitive decline (DECO score $<33$ ) ( $n$ 598) } & \multicolumn{5}{|c|}{ Functional impairment (4-IADL score $>0)(n 716)$} \\
\hline & & & \multicolumn{2}{|c|}{ Group 2 v. group $1 \dagger$} & \multicolumn{2}{|c|}{ Group 3 v. group $1 \dagger$} & \multirow[b]{2}{*}{$P$ for trend } & \multicolumn{2}{|c|}{ Group 2 v. group $1 \dagger$} & \multicolumn{2}{|c|}{ Group 3 v. group $1 \dagger$} & \multirow[b]{2}{*}{$P$ for trend } \\
\hline & & & OR & $95 \% \mathrm{Cl}$ & OR & $95 \% \mathrm{Cl}$ & & OR & $95 \% \mathrm{Cl}$ & OR & $95 \% \mathrm{Cl}$ & \\
\hline Energy (except energy from alcohol) $(\mathrm{kJ})$ & 8343 & 2232 & 0.93 & $0.75,1.15$ & 1.00 & $0.81,1.24$ & 0.957 & 0.87 & $0.71,1.06$ & 0.91 & $0.75,1 \cdot 11$ & 0.367 \\
\hline Alcohol $(\mathrm{g}) \S$ & $10 \cdot 43$ & 12.96 & 0.86 & $0.66,1.11$ & 0.84 & $0.65,1.09$ & 0.264 & 0.78 & $0.61,0.98$ & 0.76 & $0.59,0.96$ & 0.051 \\
\hline Total carbohydrates (g) & 228.5 & 74.47 & $1 \cdot 15$ & $0.92,1.42$ & 1.05 & $0.84,1.30$ & 0.696 & 0.93 & $0.75,1.14$ & $1 \cdot 12$ & $0.92,1.37$ & 0.266 \\
\hline Mono- and disaccharides (g) & $108 \cdot 2$ & 38.80 & $1 \cdot 11$ & $0.89,1.37$ & 0.94 & $0.75,1.17$ & 0.579 & 0.91 & $0.74,1 \cdot 11$ & 0.84 & $0.69,1.03$ & 0.092 \\
\hline Starch $(g)$ & $120 \cdot 3$ & 52.75 & 1.06 & $0.85,1.32$ & $1 \cdot 11$ & $0.90,1.38$ & 0.327 & 0.99 & $0.81,1.22$ & $1 \cdot 18$ & $0.96,1.44$ & 0.106 \\
\hline Soluble dietary fibre $(\mathrm{g})$ & $5 \cdot 233$ & 1.685 & 0.90 & $0.73,1.11$ & 0.74 & $0.60,0.92$ & 0.006 & 0.79 & $0.65,0.97$ & 0.86 & $0.70,1.05$ & 0.126 \\
\hline Total dietary fibre $(\mathrm{g})$ & $24 \cdot 80$ & 8.029 & 0.83 & $0.67,1.03$ & 0.79 & $0.64,0.98$ & 0.033 & 0.93 & $0.76,1.13$ & 0.89 & $0.72,1.08$ & 0.236 \\
\hline Proteins $(\mathrm{g})$ & $87 \cdot 70$ & 24.55 & 0.99 & $0.80,1.22$ & 0.92 & $0.74,1.14$ & 0.429 & 1.02 & $0.84,1.25$ & 0.84 & $0.68,1.03$ & 0.095 \\
\hline Total lipids (g) & $81 \cdot 20$ & 25.83 & $1 \cdot 17$ & $0.94,1.45$ & 1.03 & $0.83,1.28$ & 0.818 & 1.00 & $0.82,1.22$ & 0.98 & $0.80,1.20$ & 0.824 \\
\hline SFA (g) & $32 \cdot 28$ & 12.02 & 0.99 & $0.80,1.23$ & 1.02 & $0.82,1.26$ & 0.886 & 0.93 & $0.76,1.14$ & 0.89 & $0.73,1.09$ & 0.269 \\
\hline MUFA (g) & 28.54 & 9.740 & $1 \cdot 16$ & $0.93,1.44$ & $1 \cdot 16$ & $0.93,1.44$ & 0.199 & 1.06 & $0.87,1.30$ & 1.03 & $0.84,1.26$ & 0.798 \\
\hline PUFA (g) & 13.99 & 5.920 & $1 \cdot 14$ & $0.92,1.42$ & 1.04 & $0.84,1.30$ & 0.703 & 1.04 & $0.85,1.27$ & 1.06 & $0.87,1.30$ & 0.565 \\
\hline$n-6$ Fatty acids $(\mathrm{g})$ & 12.55 & 5.614 & $1 \cdot 11$ & $0.90,1.37$ & 1.03 & $0.83,1.28$ & 0.794 & 1.04 & $0.85,1.28$ & 1.03 & $0.84,1.26$ & 0.768 \\
\hline$n-3$ Fatty acids (g) & 1.419 & 0.558 & 0.91 & $0.74,1.13$ & 0.79 & $0.63,0.98$ & 0.029 & 0.84 & $0.69,1.03$ & 0.94 & $0.77,1.15$ & 0.573 \\
\hline$\alpha$-Linolenic acid $(\mathrm{g})$ & 0.926 & 0.354 & 0.95 & $0.77,1 \cdot 18$ & 0.90 & $0.73,1.12$ & 0.341 & 0.99 & $0.80,1 \cdot 21$ & 0.97 & $0.79,1 \cdot 18$ & 0.756 \\
\hline Long-chain $n-3$ fatty acids (g) & 0.488 & 0.345 & 0.95 & $0.77,1.17$ & 0.85 & $0.68,1.05$ & 0.134 & 0.85 & $0.70,1.04$ & 0.95 & $0.78,1.16$ & 0.596 \\
\hline$n-6: n-3$ Fatty acids ratio & 9.383 & 4.113 & 1.08 & $0.87,1.34$ & 1.25 & $1.01,1.55$ & 0.041 & $1 \cdot 10$ & $0.90,1.35$ & 1.09 & $0.89,1.33$ & 0.429 \\
\hline$\beta$-Carotene $(\mu \mathrm{g})$ & 4188 & 1762 & 0.94 & $0.76,1 \cdot 16$ & 1.02 & $0.82,1.26$ & 0.882 & 0.87 & $0.71,1.06$ & 0.86 & $0.71,1.05$ & 0.138 \\
\hline Retinol $(\mu \mathrm{g})$ & 1061 & 1061 & 1.46 & $1 \cdot 16,1.83$ & 1.38 & $1 \cdot 10,1 \cdot 72$ & 0.007 & 0.94 & $0.77,1.16$ & 0.91 & $0.74,1.11$ & 0.349 \\
\hline Vitamin $B_{1}(\mathrm{mg})$ & 1.256 & 0.379 & 0.87 & $0.70,1.08$ & 0.93 & $0.75,1.15$ & 0.477 & 0.82 & $0.67,1.00$ & 0.96 & $0.79,1.17$ & 0.680 \\
\hline Vitamin $B_{2}(\mathrm{mg})$ & $2 \cdot 142$ & 0.706 & 1.02 & $0.82,1.27$ & 1.05 & $0.85,1.30$ & 0.649 & 0.86 & $0.70,1.05$ & 0.80 & $0.65,0.98$ & 0.028 \\
\hline Vitamin $B_{6}(\mathrm{mg})$ & 1.762 & 0.481 & 0.84 & $0.68,1.04$ & 0.82 & $0.66,1.02$ & 0.066 & 0.91 & $0.75,1.11$ & 0.80 & $0.65,0.98$ & 0.032 \\
\hline Folic acid $(\mu \mathrm{g})$ & 401.4 & $117 \cdot 0$ & $1 \cdot 18$ & $0.95,1.46$ & $1 \cdot 12$ & $0.90,1.39$ & 0.305 & 0.87 & $0.71,1.06$ & 0.86 & $0.71,1.05$ & 0.140 \\
\hline Vitamin $B_{12}(\mu \mathrm{g})$ & $7 \cdot 713$ & 4.813 & 1.02 & $0.82,1.26$ & 1.05 & $0.85,1.30$ & 0.643 & 0.71 & $0.58,0.87$ & 0.79 & $0.65,0.97$ & 0.022 \\
\hline Vitamin C (mg) & $144 \cdot 3$ & 61.76 & 0.98 & $0.79,1.21$ & 0.81 & $0.66,1.01$ & 0.065 & 0.85 & $0.69,1.03$ & 0.86 & $0.71,1.05$ & 0.134 \\
\hline Vitamin $D(\mu \mathrm{g})$ & 2.466 & 1.306 & 0.88 & $0.71,1.09$ & 0.89 & $0.72,1 \cdot 10$ & 0.280 & $1 \cdot 10$ & $0.90,1.35$ & 1.02 & $0.83,1.25$ & 0.848 \\
\hline Vitamin $E(m g)$ & 13.61 & 5.663 & $1 \cdot 11$ & $0.89,1.37$ & 1.03 & $0.83,1.28$ & 0.806 & $1 \cdot 18$ & $0.97,1.44$ & 1.04 & $0.85,1.28$ & 0.709 \\
\hline $\mathrm{Ca}(\mathrm{mg})$ & 1026 & 403.6 & 1.26 & $1.01,1.57$ & $1 \cdot 17$ & $0.94,1.46$ & 0.174 & 0.97 & $0.80,1 \cdot 19$ & 0.90 & $0.74,1 \cdot 11$ & 0.320 \\
\hline $\mathrm{Fe}(\mathrm{mg})$ & 13.41 & 3.701 & 0.97 & $0.79,1.20$ & 0.90 & $0.72,1.12$ & 0.338 & 1.06 & $0.87,1.29$ & 0.95 & $0.78,1.17$ & 0.652 \\
\hline $\mathrm{Mg}(\mathrm{mg})$ & $392 \cdot 2$ & $122 \cdot 7$ & 0.89 & $0.72,1.09$ & 0.84 & $0.68,1.04$ & 0.105 & 1.04 & $0.85,1.26$ & 0.88 & $0.72,1.08$ & 0.228 \\
\hline$P(\mathrm{mg})$ & 1360 & 406.5 & 0.99 & $0.80,1.22$ & 0.97 & $0.78,1.21$ & 0.793 & 0.95 & $0.77,1.15$ & 0.83 & $0.68,1.02$ & 0.077 \\
\hline $\mathrm{Mn}(\mathrm{mg})$ & 3.863 & 6.531 & 0.98 & $0.77,1.24$ & 1.15 & $0.92,1.44$ & 0.176 & 0.88 & $0.71,1.09$ & 1.02 & $0.83,1.26$ & 0.701 \\
\hline$I(\mu \mathrm{g})$ & $145 \cdot 5$ & 46.87 & $1 \cdot 17$ & $0.94,1.45$ & 0.98 & $0.78,1 \cdot 21$ & 0.819 & 1.04 & $0.85,1.27$ & 0.95 & $0.78,1.17$ & 0.632 \\
\hline
\end{tabular}

DECO, 'Détérioration Cognitive Observée' (observed cognitive deterioration) questionnaire.

* Through multi-adjusted logistic regression models, taking healthy elderly as the reference group. Adjustment was made on all covariates listed in Table 1. Each nutrient was considered separately. † Group 1, group 2 and group 3 were defined as tertile 1, tertile 2 and tertile 3 of intake residuals on energy (except for alcohol).

$\S$ Because of the non-negligible percentage of abstainers and the specificity of this group, the population was split into abstainers $(13.8 \%), 0<$ mean intake $\leq 8.3 \mathrm{~g} / \mathrm{d}(43.5 \%)$ and mean intake $>8.3 \mathrm{~g} / \mathrm{d}(42.7 \%)$. 


\section{Interactions, sensitivity analysis and alternate analysis}

We verified that modification of cut-off points for adjustment variables had no effect on the final results. For example, the 80-year cut-off point was chosen for age for easier comparison, as it is a symbolic threshold, but use of 78 years (the median value), as the cut-off did not substantially modify the overall findings, nor did any sensitivity analysis using different cut-off values.

We tested potential interactions between dietary intakes and age or education level, but none yielded significant differential effects.

In order to test the stability of the present results, we used a more restrictive definition of cognitive decline using a cut-off point of 31 rather than 33 for the DECO score, which resulted in 341 women with recent cognitive decline $v$. 4468 control women. Associations between informant-appraised cognitive status and dietary intake remained quite stable for most nutrients. However, the association with $n-3$ fatty acids was no longer statistically significant, even though this nutrient still displayed a similar inverse association with cognitive decline (OR 0.93 (95\% CI $0.70,1.22)$ and 0.88 (95\% CI $0.67,1 \cdot 15$ ) for tertiles 2 and 3 respectively, taking the first tertile of intake as reference).

To rule out a possible confounding effect of dietary supplement use, we conducted our analyses in a restricted sample ( $n$ 3347), excluding all women who declared taking at least one type of supplement. Associations involving lower intakes of vitamin $\mathrm{C}$ became statistically significant with higher odds of both cognitive decline and functional impairment. No other difference was found in this subsample compared with those obtained for the whole sample (data not tabulated).

Furthermore, we tested the stability of the present results when including all women with available dietary data: women with extreme values for the energy intake:energy requirement ratio were reintegrated in the sample analysis ( $n$ 4922). The set of significant associations remained unchanged in this larger sample. For example, regarding soluble dietary fibre and DECO score $<33$ ( $n 622$ cases in this larger sample), OR for the second and third tertiles of intake ( $v$. the first tertile) were respectively 0.92 (95\% CI 0.75 , $1.13)$ and 0.76 (95\% CI 0.61, 0.94), as compared with women with a high DECO score.

\section{Discussion}

In 2006, elderly women participating in the E3N cohort that were reported by informants to have undergone recent cognitive decline had, 13 years previously, lower intakes of poultry, fish, and animal fats, as well as higher intakes of dairy desserts and ice-cream. They had lower habitual intakes of dietary fibre and $n-3$ fatty acids, but a higher intake of retinol. Furthermore, elderly women that were reported by informants to be functionally impaired had, in the past, lower intakes of vegetables and vitamins $\mathrm{B}_{2}, \mathrm{~B}_{6}$ and $\mathrm{B}_{12}$.

The main interest of the present study lies in the time interval (more than a decade) between dietary assessment and cognitive and functional assessment, which enabled us to explore the long-term effect of dietary habits in ageing. Although diet is likely to vary throughout life, our hypothesis is that it remains quite stable after menopause and retirement, but before the advanced state of ageing processes. Because these women were aged 62-68 years at the time of dietary assessment, nutritional data considered in the present study seem to be informative when relating long-term individual dietary habits to late-life outcome. Moreover, the nutritional data of the $\mathrm{E} 3 \mathrm{~N}$ cohort have provided meaningful results when studying associations between usual dietary intake and cancer occurrence, a disease that may also have a latency period of many years before being diagnosed ${ }^{(6,18)}$. Finally, the lifecourse approach to age-related disorders provided opportunities for identifying the nature and timing of environmental contributions $^{(19)}$, which is not possible in a short-term design study.

Another strength of the present study lies in the availability of extensive adjustment data. Careful control for potential confounders is important in an observational setting so as to limit biases that may arise, since socio-economic status and other behavioural and medical characteristics may influence both dietary intake and cognitive function. In the present study, we were able to implement fully adjusted models. In particular, the association between dietary intake and cognitive decline remained significant after controlling for various vascular factors (hypertension, hypercholesterolaemia, diabetes mellitus, CHD and stroke), suggesting that diet may influence cognitive ageing through pathways that are partly separate from cardiovascular processes.

In this large epidemiological study, cognitive assessment was based on informant self-response to validated questionnaires (IADL and DECO) rather than face-to-face interviews. Substantial data support the validity of using both functional and informant measures in ageing studies ${ }^{(20)}$. More precisely, DECO, which is independent of socio-economic background ${ }^{(10)}$, has been recently reported to be a good screening device for evaluating cognitive decline due to multiple causes $^{(21)}$. In addition, the 4-IADL score has proven to be a valid indicator of cognitive loss and is highly predictive of early dementia ${ }^{(11)}$. Descriptive analyses showed relationships between our outcomes of interest and characteristics usually associated with cognitive and functional impairment (age, education level, history of stroke, etc), thus supporting our definitions for testing hypotheses in the research field on age-related decline.

Outcome information analysed in the present study was informant-reported, perhaps leading to some misclassification, but there is no reason to suspect differential errors.

Missing values in dietary data or in cognitive information may lead to a selection of the study sample. Indeed, exclusion of women with non-available dietary data resulted in over-representation of younger and more educated women. These observations are consistent with the hypothesis that nonresponders are more likely to undergo adverse outcomes or to be less health-conscious ${ }^{(22)}$. Such a selection may influence study results in two ways: by decreasing the statistical power and by masking some of the effects of nutritional intake, since the cognitive impact of some nutritional deficiencies may have been underestimated because of an insufficient range of exposure in the selected analysis sample. In particular, several epidemiological studies found an association with fruit consumption, vitamin $\mathrm{C}$, vitamin $\mathrm{E}$ or $\beta$-carotene intake, in agreement with the antioxidant hypothesis. In our sample, 
we observed only a borderline association between cognitive decline and lower vitamin $\mathrm{C}$ intake. The limited range of intake in our population, with few women having low consumption levels in foods rich in antioxidant components, may explain the absence of certain associations in the present study. For example, over $95 \%$ of the elderly E3N participants consumed more than one $80 \mathrm{~g}$ portion of fruit daily, with a median intake of $350 \mathrm{~g} / \mathrm{d}$. As a comparison, the fruit consumption level was quite lower in another French cohort showing a significant protective association involving fruit and dementia: in the 'three-City cohort' study, more than $20 \%$ of the participants ate less than one daily serving of fruit ${ }^{(23)}$.

Another limitation is that our sample includes only women, which precludes generalising the present results to men, who have on the whole different food intake and nutritional status $^{(24)}$.

Our findings of significant associations between age-related decline and lower long-term intake of certain specific nutrients - namely $n$ - 3 fatty acids, dietary fibre and vitamin $\mathrm{B}_{6}$ - have already been described, generally in studies with shorter prospective design ${ }^{(25)}$. However, the present study is not in complete agreement with previous ones in that it shows that higher intake of retinol and lower intake of animal fats were both associated with cognitive decline. Potential explanations for these unexpected results include confounding effects and chance resulting from multiple analyses. Regarding residual confounding, a null intake of animal fats could be, for instance, an indicator of poor general health (similar to alcohol abstinence), thus explaining that a low intake of animal fats was related to an increased OR of cognitive decline. Moreover, in the studied sample, $70 \%$ of animal fats was butter and intake level was generally low, with $95 \%$ of the studied women consuming less than $20 \mathrm{~g} / \mathrm{d}$ (median intake $6 \mathrm{~g} / \mathrm{d}$ ). As a comparison, $90 \%$ of the population included in the Chicago Health and Aging Project consumed more than $16 \mathrm{~g}$ animal $\mathrm{fat} / \mathrm{d}^{(26)}$. This latter study concluded that a diet high in saturated fat might be associated with cognitive decline among older individuals. The intake range in the $\mathrm{E} 3 \mathrm{~N}$ population could thus be below the level where saturated fat becomes deleterious.

Regarding chance due to multiple analyses, we performed nearly sixty models for each outcome of interest. Nutritional epidemiology, especially when dealing with large-scale studies such as ours, is restricted by both the fact that multiple analyses are performed (often leading to separate reports for each category of foods or nutrients), and that misclassification of the diet results in a decreased power of the studies ${ }^{(27)}$. Thus very few, if any, use the Bonferroni correction, which would most often lead to no significant association at all. Restricting analyses to variables already described in the literature, such as vitamins and fatty acids, would limit the opportunity to explore novel hypotheses, although more emphasis should be made on findings consistent with the literature. New findings, such as the association with retinol, need to be further confirmed.

Several neuropathogenic mechanisms can be evoked as underlying biological processes for the observed associations. $n-3$ Fatty acids, in which fish is rich, act on heart and brain not only through the vascular pathway but also through different cellular mechanisms: heart rhythm; neurotransmission; neuroprotection; neurogenesis. Similarly, some B vitamins and homocysteine can act directly on brain cell functioning. Anti-inflammatory and antioxidant properties are suggested to account for the inverse association with dietary fibre and vegetables. On the whole, a number of experimental studies, and a variety of partly common mechanisms, support a favourable effect of these compounds.

Although underlying biological pathways are not yet fully elucidated, the present study nonetheless has public health implications along with previous studies ${ }^{(28)}$. The present results suggest that prevention of age-related impairment may be reached through a balanced diet rich in vegetables, fish and poultry, and limited in sweet dairy products, not only in later life but starting in middle age. These recommendations, also suggested for cancer ${ }^{(29)}$ and cardiovascular protection $^{(30)}$, may enhance the quality of life ${ }^{(31)}$.

Our large-scale longitudinal study with validated dietary data may represent a valuable contribution to a better understanding of the link between long-term nutrition and latelife cognition. The present results support the hypothesis that high intakes of $n-3$ fatty acids, dietary fibre and some B-group vitamins may contribute to a reduction in agerelated impairment.

\section{Acknowledgements}

The E3N cohort is supported by the French League against Cancer, the European Community, 3M Company, Mutuelle Générale de l'Education Nationale (MGEN), French Institute of Health and Medical Research, Gustave Roussy Institute and several general councils in France. M.-N. V. receives a grant from the Statlife Company and the 'Association Nationale de la Recherche Technique'. None of the funding agencies played a role in the design or conducting of the study, analysis or interpretation of data, nor in preparation and approval of the manuscript.

F. C.-C. is the principal investigator of the E3N Cohort Study and took responsibility for the integrity of the data. M.-N. V. conducted the statistical analysis, interpreted the data and drafted the manuscript. M.-C. B.-R., K. R., F. C.-C. and C. B. contributed to the writing and revisions. C. B. supervised the statistical analyses. All authors critically reviewed the manuscript.

The authors report no conflict of interest.

\section{References}

1. McGuire LC, Ford ES \& Ajani UA (2006) Cognitive functioning as a predictor of functional disability in later life. Am J Geriatr Psychiatry 14, 36-42.

2. Blennow K, de Leon MJ \& Zetterberg H (2006) Alzheimer's disease. Lancet 368, 387-403.

3. Gillette-Guyonnet S, Van Kan GA, Andrieu S, et al. (2007) IANA task force on nutrition and cognitive decline with aging. J Nutr Health Aging 11, 132-152.

4. Ortega RM, Requejo AM, Andres P, et al. (1997) Dietary intake and cognitive function in a group of elderly people. Am J Clin Nutr 66, 803-809.

5. Gonzalez-Gross M, Marcos A \& Pietrzik K (2001) Nutrition and cognitive impairment in the elderly. Br J Nutr 86, 313-321. 
6. Touillaud MS, Thiebaut AC, Fournier A, et al. (2007) Dietary lignan intake and postmenopausal breast cancer risk by estrogen and progesterone receptor status. J Natl Cancer Inst 99, 475-486.

7. Katz S (1983) Assessing self-maintenance: activities of daily living, mobility, and instrumental activities of daily living. $J$ Am Geriatr Soc 31, 721-727.

8. Ritchie K \& Fuhrer R (1992) A comparative study of the performance of screening tests for senile dementia using receiver operating characteristics analysis. $J$ Clin Epidemiol 45, 627-637.

9. Ferrari P, Slimani N, Ciampi A, et al. (2002) Evaluation of under- and overreporting of energy intake in the 24-hour diet recalls in the European Prospective Investigation into Cancer and Nutrition (EPIC). Public Health Nutr 5, 1329-1345.

10. Ritchie K \& Fuhrer R (1996) The validation of an informant screening test for irreversible cognitive decline in the elderly: performance characteristics within a general population sample. Int J Geriatr Psychiatry 11, 149-156.

11. Barberger-Gateau P, Fabrigoule C, Helmer C, et al. (1999) Functional impairment in instrumental activities of daily living: an early clinical sign of dementia? J Am Geriatr Soc 47, 456-462.

12. van Liere MJ, Lucas F, Clavel F, et al. (1997) Relative validity and reproducibility of a French dietary history questionnaire. Int J Epidemiol 26, Suppl. 1, S128-S136.

13. Lucas F, Niravong M, Villeminot S, et al. (1995) Estimation of food portion size using photographs: validity, strength, weaknesses and recommendations. J Hum Nutr Diet 8, 65-74.

14. Favier JC, Ireland-Ripert J, Toque C, et al. (1995) Répertoire Général des Aliments. Table de Composition (Food Composition Table). Paris: TEC \& DOC.

15. Fratiglioni L, Paillard-Borg S \& Winblad B (2004) An active and socially integrated lifestyle in late life might protect against dementia. Lancet Neurol 3, 343-353.

16. Silverman BW (1986) Density Estimation. London: Chapman and Hall.

17. Willett WC, Howe GR \& Kushi LH (1997) Adjustment for total energy intake in epidemiologic studies. Am J Clin Nutr 65, $1220 \mathrm{~S}-1228 \mathrm{~S}$.
18. Touvier M, Kesse E, Clavel-Chapelon F, et al. (2005) Dual association of $\beta$-carotene with risk of tobacco-related cancers in a cohort of French women. J Natl Cancer Inst 97, 1338-1344.

19. Whalley LJ, Dick FD \& McNeill G (2006) A life-course approach to the aetiology of late-onset dementias. Lancet Neurol 5, 87-96.

20. Tierney MC, Herrmann N, Geslani DM, et al. (2003) Contribution of informant and patient ratings to the accuracy of the mini-mental state examination in predicting probable Alzheimer's disease. J Am Geriatr Soc 51, 813-818.

21. Cullen B, O’Neill B, Evans JJ, et al. (2007) A review of screening tests for cognitive impairment. J Neurol Neurosurg Psychiatry 78, 790-799.

22. Goldberg M, Chastang JF, Zins M, et al. (2006) Health problems were the strongest predictors of attrition during followup of the GAZEL cohort. J Clin Epidemiol 59, 1213-1221.

23. Barberger-Gateau P, Raffaitin C, Letenneur L, et al. (2007) Dietary patterns and risk of dementia: the Three-City cohort study. Neurology 69, 1921-1930.

24. Hercberg S, Czernichow S \& Galan P (2006) Antioxidant vitamins and minerals in prevention of cancers: lessons from the SU.VI.MAX study. Br J Nutr 96, Suppl. 1, S28-S30.

25. Luchsinger JA \& Mayeux R (2004) Dietary factors and Alzheimer's disease. Lancet Neurol 3, 579-587.

26. Morris MC, Evans DA, Bienias JL, et al. (2004) Dietary fat intake and 6-year cognitive change in an older biracial community population. Neurology 62, 1573-1579.

27. Willett W (1998) Nutritional Epidemiology. New York, Oxford: Oxford University Press.

28. Luchsinger JA, Noble JM \& Scarmeas N (2007) Diet and Alzheimer's disease. Curr Neurol Neurosci Rep 7, 366-372.

29. World Cancer Research Fund \& American Institute for Cancer Research (2007) Nutrition, Physical Activity and the Prevention of Cancer: a Global Perspective. Washington, DC: AICR.

30. De Caterina R, Zampolli A, Del Turco S, et al. (2006) Nutritional mechanisms that influence cardiovascular disease. Am J Clin Nutr 83, 421S-426S.

31. Kennedy ET (2006) Evidence for nutritional benefits in prolonging wellness. Am J Clin Nutr 83, 410S-414S. 